\title{
TEORÍA Y PRÁCTICA DE LA INTERVENCIÓN EN CRISIS: UNA PROPUESTA DESDE LA PSICOLOGÍA SOCIAL
}

\section{THEORY AND PRACTICE OF CRISIS INTERVENTION: A PROPOSAL FROM SOCIAL PSYCHOLOGY}

\section{ERNESTO FLORES SIERRA ${ }^{1}$}

Recibido: 15 de mayo de 2017 Aceptado: 29 de agosto de 2017

\footnotetext{
${ }^{1}$ Pontificia Universidad Católica del Ecuador, Facultad de Psicología, Quito, Ecuador (eflores293@puce.edu.ec).
} 



\title{
TEORÍA Y PRÁCTICA DE LA INTERVENCIÓN EN CRISIS: UNA PROPUESTA DESDE LA PSICOLOGÍA SOCIAL
}

\author{
THEORY AND PRACTICE OF CRISIS \\ INTERVENTION: A PROPOSAL FROM SOCIAL \\ PSYCHOLOGY
}

Ernesto Flores Sierra

Palabras clave: Psicología de la liberación, intervención psicológica en catástrofes, psicología social, psicología comunitaria.

Key Words: Libertary Psychology, psychological intervention in catastrophes, social psychology, community psychology.

\section{RESUMEN}

El artículo realiza un resumen teórico de los principales postulados de la psicología social, que también es definida como psicología política o psicología de la liberación, desarrolla los principios fundamentales del accionar de los psicólogos que se acogen a esta visión en situaciones de catástrofes o emergencias. Postula una serie de principios teóricos y técnicos que guían una intervención psicosocial pues se considera al sujeto como una unidad dialéctica entre lo personal, lo comunitario y lo social; se propone además la herramienta psicodramática como la técnica de una intervención que tenga como finalidad una perspectiva de liberación en el marco de la 
atención de la crisis psíquica que genera la fragmentación del ciclo de vida de la persona que ha sido víctima de una catástrofe.

\section{ABSTRACT}

The paper presents a theoretical summary of the postulates of social psychology, which is also defined as political psychology or libertary psychology, developing the principles of the action of psychologists in situations of catastrophes or emergencies. Postulates theoretical and technical principles that guide a psychosocial intervention considering the subject as a dialectical unit between subjectivity, community and society, also proposing the psychodramatic tools as the technique for an intervention with an libertary perspective in the attention of the psychic crisis that generates the fragmentation of the life cycle of the person who has been the victim of a catastrophe.

\section{INTRODUCCIÓN}

La psicología social, política y comunitaria constituye un enfoque desarroIlado fundamentalmente en América Latina como una respuesta a las necesidades específicas de las sociedades del subcontinente (Montero, 2004) sobredeterminadas por las condiciones estructurales de dependencia, violencia y pauperización creciente, donde las comunidades y los pueblos se han desenvuelto históricamente en un proceso de disputa con los Estados nacionales y sus políticas de control social determinadas por las necesidades de los capitales, los procesos de extracción de materias primas y los movimientos financieros internacionales.
Las propuestas de la psicología comunitaria se desprenden de la teoría crítica (escuelas marxistas rusa, de Frankfort, francesa, norteamericana), los aportes de la psicología europea (Montero, 2004) y la misma investigación- acción de psicólogos latinoamericanos como: Martín Baró, Maritza Montero, Pichon Riviere, Alfredo Moffatt, Ana P. de Quiroga, y otros, que han configurado un corpus teórico y práctico que se ha desarrollado en comunidades, barrios, hospitales psiquiátricos, centros de reclusión, etc., es decir en todos aquellos lugares donde las comunidades han emprendido procesos de dignificación de su existencia. (Moffatt, 1974) 


\section{DESARROLLO}

\section{Situaciones de catástrofe, crisis e intervención en crisis}

Las situaciones de catástrofe generan en los sujetos el aparecimiento de crisis, las mismas se manifiestan en el rompimiento del ciclo vital de la persona, lo que implica que las perspectivas de futuro, de vida, de existencia se fragmentan afectando no solo al individuo, sino a todo el grupo social al que el sujeto pertenece. Las crisis implican una paralización de la temporalidad psíquica, donde la perspectiva de existencia de las personas y comunidades se ve detenida y este estancamiento existencial detona alteraciones comportamentales y afectivas que comienzan a enfermar a la persona y a la dinámica diaria de la vida social.

La crisis se manifiesta por la invasión de una experiencia de paralización de la continuidad del proceso de la vida. De pronto nos sentimos confusos y solos, el futuro se nos aparece vacío y el presente congelado. Si la intensidad de la perturbación, sea una crisis de crecimiento (evolutiva) o la consecuencia de un cambio imprevisto (traumática), aumenta, comenzamos a percibirnos como "otro" es decir tener una experiencia de despersonalización. (Moffatt, 1982, pág. 15)
En el caso de catástrofes consecuencia de desastres naturales encontramos que la crisis que experimentan los sujetos y comunidades es desintegradora por lo imprevisto de la misma, que genera la llamada experiencia de "despersonalización". Las catástrofes implican muerte, pérdidas materiales, desaparición de fuentes de trabajo, destrucción de proyectos y perspectivas de vida, lo que significa que las comunidades que habían establecido dinámicas de vida económico- sociales y culturales, de manera imprevista ven detenida toda su experiencia de mundo, y la base psíquica se ve alterada fundamentalmente en el hecho de que el sujeto pierde la capacidad de poner en movimiento sus herramientas psíquicas (Vygotski, 1979/2009) experimentando la situación de inmovilidad, inhibición y miedo.

El estado que se desarrolla por la situación antes descrita ha sido definido como "angustia", el sujeto ante el recuerdo de la catástrofe o circunstancias que le hacen rememorar lo sucedido (como las réplicas de un sismo) experimenta lo que los manuales de diagnóstico han denominado "crisis de angustia" o "ataque de pánico", durante los cuales, la persona experimenta sensación de falta de aire, hiperhidrosis, dolor en el pecho, temor a la muerte, temor a volverse "loco", 
etc., síntomas que generan un grave agotamiento corporal que lleva al sujeto a la evitación, el miedo, el solipsismo y el estado permanente de angustia generalizada. La intervención de emergencia, se encarga de atender específicamente estos casos, y la metodología de acción procura evitar que dicho estado agudo reactivo a la catástrofe se convierta en un estado crónico que se integre al sistema de personalidad del sujeto desorganizando permanentemente su conducta.

La expresión orgánica de este proceso de desorganización de la personalidad es la angustia vivida corporalmente, que se acompaña de trastornos cardio- respiratorios, ahogos y la sensación de tener "nudos" en el estómago y la garganta (...) se trata de un estado de estres o sensación de agotamiento corporal que padece la persona en crisis. (...) Lo que enferma, pues, en el estado de crisis es el proceso de vivir, la historia se discontinúa y, por lo tanto, el yo no puede percibirse como una sucesión inteligible y se fractura sin atinar a concebir su nueva situación (a codificarla) y sin saber cómo actuar, pues las estrategias con que contaba ya no se adaptan a las nuevas circunstancias. (Moffatt, 1982, pág. 16)
Esta angustia es la manifestación sintomática de un estado de crisis en el proceso de vida de la persona, es una experiencia en la cual los parámetros cotidianos de existencia (casa, familia, trabajo, rutina, perspectivas) se ven reducidas, destruidas, fragmentadas, $y$, se vuelven inteligibles, imposibles, irrealizables para el mismo sujeto. La nueva situación provocada por la catástrofe es difícil de asimilar y la imposibilidad de pensar la situación implica la imposibilidad de accionar sobre la misma, las estrategias vitales construidas en el proceso de vida y educación del sujeto no responden a la circunstancia inesperada y el sujeto cae paulatinamente en un estado de alteración grave del comportamiento, que muchas veces se encubre por la inactividad propia de la vida en los albergues.

La intervención en esta etapa busca abrir espacios para que el sujeto pueda asimilar, pensar y verbalizar la nueva e inesperada circunstancia, y de esta manera, genere herramientas para poder actuar sobre la misma; herramientas que aparecen en las relaciones interpersonales que la nueva circunstancia obliga, y que paulatinamente la persona internaliza convirtiéndolas en funciones intrapsíquicas. El reconocimiento de la situación nueva implica la posibilidad de desarrollar alternativas de afrontamiento que además permiten que el sujeto movilice los afectos contenidos que se 
habían estancado manifestándose como crisis de angustia frente a la situación inesperada y se produzca el proceso de adaptación a las nuevas condiciones materiales. El psicólogo permite y abre espacio para que el sujeto hable sobre lo vivido para de esta manera ordenar sus ideas, pensamientos, miedos, sufrimientos relacionados con la experiencia, y en ese hablar se reconstruyan las herramientas psíquicas fragmentadas por la catástrofe, permitan además que el sujeto se reintegre y desarrolle nuevas estrategias de afrontamiento frente a las nuevas y desconocidas circunstancias.

\section{Psicoterapia del oprimido, psicología política y atención de emergencia}

Las situaciones de crisis como consecuencia de catástrofes afectan fundamentalmente a los sectores históricamente empobrecidos de la población puesto que sus condiciones materiales de existencia los vuelven más vulnerables a este tipo de eventos (en el caso de los terremotos, por ejemplo, la mayoría de viviendas que colapsan suelen ser viviendas construidas de manera precaria donde viven hacinadas personas de sectores pauperizados, o en el caso de las inundaciones, donde las viviendas arrasadas son de personas que por su empobrecimiento se han asentado en los lechos de los ríos), esto implica que la acción del psicólogo en el campo en situaciones de desastres puede enmarcarse en el ámbito de la psicología política, es decir enfocando la problemática psíquica como una problemática que se desprende de las condiciones materiales de explotación, empobrecimiento y marginalización de estos sectores.

Por lo tanto, la intervención parte del análisis de los mundos de vida de los sujetos con quienes se realiza la intervención, procurando identificar los aspectos culturales y sociales de las personas que se encuentran en situación de crisis. El psicólogo comunitario considera todos los elementos centrales de la vida comunitaria. Esta consideración evita enajenar al sujeto en crisis de sus propias necesidades y someterlo a la visión externa del psicólogo u operario de salud mental.

Las necesidades de las colectividades se encuentran determinadas por su proceso de constitución histórico y social, y este proceso generalmente es un proceso sobredeterminado por las condiciones económicas generales de la sociedad a la que las personas pertenecen, que a su vez responde a la formación económico- social global en la que todos nos encontramos en un momento histórico determinado. Esta triple implicación genera necesidades propias que en los momentos de las catástrofes se ven o bien pospuestas por la situación 
de emergencia (necesidades de acceso a la educación o perspectivas de promoción social), o bien potenciadas por las consecuencias mismas de la calamidad (alimentos, vivienda, salud), y que al verse desintegrado el ciclo de vida del sujeto, se presentan psíquicamente como irresolubles e inalcanzables, y generan la ansiedad y malestar personal descritos en el apartado anterior. Estos fenómenos son parte de un complejo proceso social, y por lo tanto, su solución es un problema fundamentalmente social y colectivo, y es necesario evitar que el sujeto los afronte como problemas individuales, puesto que al hacerlo genera una sobrecarga de síntomas sobre el sujeto que los presenta, pues este pasa a convertirse en el "sujeto patológico" del sistema comunitario.

La propuesta de la psicología política, propone que si los fenómenos de alteración psíquica se originan en el marco social, la solución a los mismos, y la reorientación del sujeto a su condición de agente de transformación comunitaria, es un proceso social y necesita trabajarse bajo el principio de la llamada "redistribución de la locura". Esta posibilidad de permitir que el grupo comunitario afronte la problemática personal como un problema social, implica desarrollar el vínculo comunitario del empoderamiento, es decir, asumir la ansiedad que aparece en los miembros afectados por el desastre como un problema de todos, y por lo tanto la única forma de solucionarlo es mediante la comunión, el diálogo y la construcción de respuestas comunitarias actuando como elemento terapéutico central. Al lograr esto, la identidad del paciente en relación con su comunidad puede regenerarse en función de sus roles comunitarios, es decir, el grupo comunitario permite el rencuentro del sujeto consigo mismo y con su propia historia. Asumir de manera colectiva la crisis y el sufrimiento, brinda al sujeto un soporte comunitario que le permite desarrollar su subjetividad en el marco del encuentro con otros que han sufrido situaciones similares y que comparten su situación de crisis psíquica. (Moffatt, 1974)

La identidad del paciente es recuperada mediante el reconocimiento por sí mismo y por los otros de su rol social, al ser reconocido puede ver su acción dentro de la comunidad como necesaria e importante y redignificar su propia vida en relación con los demás. Al recuperar su historia y su acción, el tiempo del sujeto vuelve a organizarse en función de resolver sus necesidades personales-comunitarias desarrollando políticamente su acción, entendiendo la política como la acción de un grupo social para defender sus intereses en el marco de una sociedad clasista. 
Por lo mismo, aparece un aquello que la psicología política define como "motor del cambio terapéutico"

Esto también hace que el "motor" del cambio terapéutico esté más que todo en la reivindicación de justicia del pueblo marginado y, por lo tanto, solo reinterpretando las técnicas psicoterapéuticas desde las modalidades de vida de nuestro pueblo, sus valores, sus mitos, su folclore, es que se va a poder operar una verdadera cura respecto de nuevos criterios de salud mental e insertado en el proceso de liberación, que es la "terapia" para la otra enfermedad, la pobreza, resultado de la explotación social y la degradación material. (Moffatt, 1974)

Las situaciones de catástrofe una vez que el núcleo comunitario se ve fortalecido por la respuesta colectiva al problema personal, generan la necesidad de una respuesta política de la comunidad afectada, la misma que demanda una reivindicación por parte del Estado que es quien tiene que responder por la afectación sufrida, y esto implica que el fortalecimiento comunitario refuerza el antagonismo comunidad- Estado, generando una lucha donde las herramientas culturales, como los mitos, el folclore, las prácticas comunitarias, fortalecen el elemento comunal que demanda justicia y respuestas eficientes frente a las tragedias acontecidas.

La salud mental, desde la perspectiva de la psicología política, busca que la comunidad una vez que ha permitido que el sujeto reconstruya su herramienta psíquica, reconstruya sus herramientas comunitarias como un refuerzo fundamental en sus demandas y dialéctica con el aparato estatal. Es por esto que la acción terapéutica es una acción liberadora que asume una postura por la comunidad, que desarrolla la práctica terapéutica en el marco de estas necesidades de acción y fortalecimiento de los pueblos y sus capacidades, no es una práctica neutral, apolítica, distanciada de la realidad, sino una acción concreta de transformación social.

Estas particularidades de la intervención en crisis desde la psicología política demandan que la metodología de intervención no parta desde una visión colonial del tratamiento, sino que reconstruya sus criterios de acción-intervención evitando negar o desconocer los marcos de existencia cultural de los pueblos afectados. Esto implica que el psicólogo debe sumergirse en estos parámetros culturales y establecer un diálogo de saberes que permita llegar a alternativas de salud nacidas de la misma comunidad.

En sociedades con fuertes rezagos feudales como las sociedades andinas 
(Cueva, Mariátegui), las relaciones nacidas del régimen de hacienda determinan las relaciones sociales entre los habitantes de las mismas, por lo cual, el momento en que el psicólogo no logra establecer el diálogo de saberes, reproduce la dinámica de "hacienda" donde el "señor que ordena" descrito por Moffatt (Moffatt, 1974), se convierte en "Patrón", quien reproduce el sometimiento feudal que dio origen a las comunidades empobrecidas. La actitud dialógica y democrática del psicólogo, por el contrario, enriquece la dignificación de los sectores empobrecidos y fortalece su acción contra su condición de opresión. En este punto la psicología política retoma los postulados de Paulo Freire que en Pedagogía del oprimido, propone que no se pueden establecer relaciones de liberación si antes no se considera la dignidad del otro, y una vez reconocida esta se permite que ese otro se convierta en el agente fundamental de su propio proceso de transformación social.

Este proceso de empoderamiento de su propia liberación construido desde las herramientas de la psicología política permite que el sujeto que ha sido víctima de la catástrofe recupere su prospectiva de tiempo, en función de los objetivos comunitarios que lo determinan como un agente de transformación social, es decir un sujeto en comunidad que construye un proyecto futuro de liberación.

\section{Psicología comunitaria e intervención psicosocial}

La psicología comunitaria es entendida como una forma de acción psicológica que se hace con la comunidad y desde la misma comunidad, esto implica que dicha actividad se diferencia del asistencialismo, puesto que la perspectiva del psicólogo comunitario no es la de "ayudar" o "resolver" los problemas de la comunidad como una acción "desde fuera", sino que persigue potenciar a la misma para encontrar autónomamente respuestas a los problemas que enfrenta, así como permitir el desarrollo de sus proyectos de liberación desde dentro. La comunidad como sujeto activo de la transformación social, es también un sujeto activo de su propia salud mental. (Montero, 2004)

La perspectiva comunitaria rescata la capacidad histórica de la comunidad para resolver sus propios problemas, reconstruye la colectividad como agente social, procurando no enajenar a la misma de su acción transformadora y reguladora. Durante las crisis provocadas por catástrofes sociales o naturales, la comunidad también se ve desintegrada por la violencia del fenómeno que trastoca también la perspectiva histórica de la misma; las movilizaciones forzadas, las pérdidas materiales, las muertes, fragmentan la acción comunitaria en el tiempo, los objetivos comunitarios se ven 
pospuestos por la tragedia y los actores tienen que concentrarse en la emergencia inmediata.

Es por estas razones que la intervención comunitaria indaga por la situación misma de la comunidad, por sus relaciones "naturales" para encontrar en las mismas las capacidades sociales para enfrentar las problemáticas desprendidas de la catástrofe y recuperar su perspectiva histórica. Esta indagación pone en debate, discusión y reflexión las propuestas de la ciencia psicológica en el seno de la comunidad. No se trata de imposiciones, sino de debates y consensos entre los procesos propios de la colectividad organizada o no organizada y el conocimiento científico del psicólogo como actor comunitario.

El reencuentro con sus propias aspiraciones es un momento de fundamental importancia en el proceso de salud mental comunitario, en el caso de desastres este encuentro implica el primer paso en el proceso de salir del estado de inhibición, de movilizarse, de reencontrarse con sus mismos procesos de transformación iniciados antes de la catástrofe y que continuarán tras la misma. Este reencuentro implica trazar líneas de acción y planificación para retomar el sentido de la acción colectiva. Este trazado, no puede ser impuesto, no existe la posibilidad de forzar a la comunidad a una acción desarrollada por intereses ex- ternos, esto implica enajenación y debilitación de la comunidad; el trazado por lo tanto debe ser autónomo y el psicólogo contribuye identificando las relaciones naturales y potenciando este proceso de redescubrimiento y fortalecimiento, que desarrollan la autonomía y el empoderamiento comunitario.

El empoderamiento permite catalizar el proceso de uso social de las potencialidades desarrolladas por la comunidad en su dinámica de enfrentamiento contra los aparatos de ejercicio del poder, el uso de las herramientas comunitarias actúa como eje de la propia recuperación frente a las circunstancias de crisis.

\begin{abstract}
El objetivo de la psicología social comunitaria es catalizar la organización y las acciones necesarias para que la comunidad use sus recursos, reconozca y emplee el poder que tiene, o bien busque otros recursos y desarrolle nuevas capacidades, generando así el proceso desde sí misma (Montero, 2006, pág. 35)
\end{abstract}

Este proceso implica un proceso en el cual la misma colectividad entra en contacto con aquellos recursos que durante su proceso de conformación consolidaron su estructura comunitaria, y que en el proceso de su devenir muchas veces pasan a quedar desapercibidos, 
estos recursos sociales, de salud, históricos, ideológicos, políticos, que accionan la vida comunitaria, necesitan pasar a ser releídos en función de las nuevas circunstancias y comprendidos en su proceso de conformación histórica.

Las catástrofes marcan puntos de quiebre en estos procesos históricos de conformación comunitaria, y los recursos construidos en la historia previa son redefinidos por el desastre y la salida del mismo, esta redefinición del mismo recurso los constituye en un nuevo recurso nacido de un doble recorrido histórico: el recorrido original donde se conformó la estructura comunitaria, y el recorrido que comienza a raíz de la tragedia, que lo termina definiendo y consolidando, puesto que su comprobación y reconstrucción en la circunstancia concreta lo integra como conocimiento comunitario y potencialidad de desarrollo e historia.

A partir de este reencuentro con su propia historia, la comunidad es capaz de adquirir un nuevo compromiso consciente con su propio devenir, este se sustenta en el desarrollo de la identidad social que se fortalece en el reencuentro y la actualización histórica de los recursos comunitarios. Esto permite que el reconocimiento histórico encuadre al sujeto en el marco de la cultura. La crisis genera la necesidad de un encuentro entre el proceso de la historia comunitaria, que define la cultura y permite la acción del sujeto.

\section{Técnicas de Intervención}

\section{Intervención psicosocial}

El primer paso de una intervención desde la perspectiva de la psicología política necesita considerar el aspecto social, es decir la condición material y comunitaria en que se encuentran las víctimas del desastre. Esta necesidad demanda un análisis concreto de las circunstancias concretas, es decir, necesita partir del estado material de las circunstancias para avanzar hacia el estado de la colectividad y terminar en el análisis del sujeto víctima. En casos de desastres, la alteración del ciclo de vida de los sujetos cambia dramáticamente su relación con el contexto, el estado de desprotección en que se sumen las colectividades altera los vínculos sociales que las personas han construido en medio de sus redes comunitarias de relación, por lo cual el primer nivel de intervención consiste en la investigación- acción que permita comprender cómo se encuentra la relación del sujeto con su entorno. (PichonRivière, 1985/ 2006)

Pichon- Riviere, en la obra citada, nos propone tres niveles para realizar este análisis, en primer lugar necesitamos observar y comprender el estado 
en que se encuentran los sujetos, si presentan o no alteraciones, cómo se encuentra su estado de ánimo, valorar si condiciones patológicas previas se han visto descompensadas por el desastre, el aparecimiento de problemáticas familiares que generan altos niveles de sufrimiento y malestar, y esto se manifiesta "hacia afuera", es decir, en la conducta del sujeto, que en casos de emergencia se refiere a su comportamiento en el albergue, en la institución, en el barrio, en la comunidad, en los lugares donde las relaciones se reconstruyen en torno a la experiencia trágica.

La investigación psicosocial analiza la parte del sujeto que se expresa hacia afuera, hacia los distintos miembros que lo rodean, en tanto que el estudio sociodinámico analiza las distintas tensiones existentes entre todos los miembros que configuran la estructura del grupo familiar dentro del cual está incluido el paciente. El análisis institucional consiste en la investigación de los grandes grupos (...) Esta triple investigación nos permite lograr un análisis completo del grupo que estamos investigando. Analizamos las tensiones del paciente con los distintos miembros del grupo, analizamos el grupo como totalidad en sí, e investigamos las funciones del intragrupo, por ejemplo, los lide- razgos. (Pichon- Rivière, 1985/ 2006, pág. 25)

En un segundo nivel, el psicólogo social analiza las relaciones sociales donde se encuentran insertos los sujetos, y analiza que dichas relaciones se encuentran atravesadas de tensiones y conflictos que dificultan el quehacer comunitario en medio de los albergues o los lugares donde los afectados reproducen su vida. Este análisis de las relaciones sociales va de la mano del análisis personal anterior, es decir observamos el comportamiento del sujeto hacia afuera, y luego observamos las relaciones que se establecen en este afuera, como las subjetividades se encuentran, chocan, confrontan, estableces consensos y disensos, y fundamentalmente cómo se articular grupos naturales sobre los cuales el psicólogo social va a reconstruir el tejido social destruido por el desastre.

Finalmente es necesario analizar la relación del grupo comunitario, en este caso el grupo de afectados por el desastre con el barrio, con la ciudad, las relaciones políticas que influyen, perjudican o ayudan a la reconstrucción de los vínculos sociales de las personas que se encuentran afectadas. Este análisis es fundamental para la intervención desde la perspectiva de la psicología social, por cuanto no se considera a los grupos sociales como entes aislados, sino como un 
parte integrante de una estructura social donde la base económico- social determina las formaciones sociales. (Cueva, 1988). Identificar estos aspectos es un ejercicio fundamentalmente práctico donde el psicólogo utilizando las herramientas de la intervención en crisis logra ingresar al grupo comunitario y convertirse en un observador participante, es decir aplica la metodología de la investigación-acción.

Esta relación entre lo personal, comunitario y social se estructura en una espiral dialéctica, lo social internalizado determina el psiquismo del sujeto como individualidad (Vygotski, 1979/2009), como explicó Vygotsky, es la condición de la formación de este continuo dialéctico entre lo social y lo personal que debe ser indagado por el psicólogo social para conseguir asentar su accionar sobre el proceso, observando los fenómenos psíquicos en su movilidad y no como fenómenos estáticos aislados de la realidad social.

Hablamos de vínculos internos y de vínculos externos integrados en un proceso de espiral dialéctica. El vínculo que primero es externo, después se hace interno y luego externo nuevamente y posteriormente vuelve a ser interno, etcétera, configurando permanentemente la fórmula de esa espiral dialéctica, de ese pasaje de lo adentro afuera y de lo afuera adentro, lo que contribuye a formar la noción de límites entre el adentro y el afuera. Esto determina que las características del mundo interno de una persona dada sean completamente diferentes de las del mundo interno de otras personas frente a la misma experiencia de la realidad externa. (Pichon- Rivière, 1985/ 2006, pág. 55)

Analizar estas particularidades permite comprender la estructuración de experiencias diversas sobre el mismo fenómeno, el triple nivel de análisis permite establecer los niveles de estudio que vayan desde lo societal hasta la experiencia personal de cada sujeto. Esta experiencia por lo mismo se encuentra construida desde una misma determinación socioeconómica, pero que por ser dialéctica la cadena del "adentro y afuera" determina formas de manifestación subjetiva, es decir, los sujetos internalizan relaciones sociales generales iguales, pero la forma como las mismas determina la vida psíquica se encuentra determinada por las condiciones históricas personales de cada persona. La experiencia de la catástrofe por lo tanto es asimilada y vivida de diferente manera por cada sujeto, el análisis propuesto por Pichon- Riviere permite leer este proceso fenomenológico sin desconocer el ciclo de espiral dialéctico que unifica lo social con lo subjetivo. 


\section{Salida del estado de inhibición- Psicodrama}

Pasados los primeros momentos de la catástrofe el sujeto que ha visto alterada su temporalidad, su clico de vida, entra en un estado de crisis de inhibición, este estado genera, como vimos en apartados anteriores, incapacidad de acción, angustia, depresión, etc., y si además consideramos, que desde la perspectiva de la psicología política es necesario que el sujeto vuelva a empoderarse de su propia historia en la dinámica general de enfrentamiento con el Estado y el capital, es fundamental que la intervención le permita a la persona recobrar su capacidad de transformar conscientemente su realidad, y enfrentar activamente las consecuencias del desastre.

El espacio de libertad que brinda el psicodrama es ese lugar donde el sujeto puede recobrar el control sobre su propia historia, donde puede recobrar su dimensión temporal, donde la acción terapéutica no se concentra en rememorar lo acontecido, sino en recobrar la capacidad de administrar sus herramientas psíquicas para controlar su comportamiento de manera consciente. Es una terapia "de la gente para la gente", donde los roles se invierten y el agente terapéutico es la misma colectividad, no el psicólogo, se emancipa al sujeto y se lo empodera de su propio devenir psíquico. Y además este reempoderamiento implica también, la modificación social del comportamiento, puesto que en la acción grupal del psicodrama el sujeto vuelve a entrar en actividad en función de la comunidad, para la comunidad, con la comunidad.

\begin{abstract}
El psicodrama define el drama como una extensión de la vida y de la acción, más bien que como su imitación, pero donde hay imitación en el énfasis no está en el que imita, sino en la oportunidad de recapitular problemas no resueltos dentro de un ambiente social más libre, más amplio y más flexible. (Moreno, 1961, pág. 39)
\end{abstract}

La redignificación que persigue la psicoterapia liberadora solo puede ser conseguida en un ambiente de libertad, la sociedad contemporánea se encuentra en un estado de desarrollo en el cual el control disciplinario abarca todos los ámbitos de la vida del sujeto (Deleuze, 1995), esto implica que el momento en que el psicólogo social abre un espacio de libertad, no solo que obtiene los beneficios terapéuticos de la actividad psicodramática, sino que permite entrar a la persona al ámbito de la recuperación de su libertad como premisa de superación de la alienación.

La postura del psicólogo desde esta perspectiva se distancia también de 
una postura directiva que impida que la comunidad recupere el flujo normal de su actividad a base de sus propias herramientas comunitarias, Moreno propondrá que "el terapeuta individual no puede transferirle automáticamente al grupo sus conocimientos y habilidades", sino que el proceso de acercamiento pasa por un complejo proceso psíquico que permite el desarrollo de la actividad psicodramática con éxito, y que en ese proceso el potencial terapéutico de los miembros del grupo se puede desarrollar convirtiéndose en la base de la técnica de recuperación del estado de actividad previo:

Uno de los momentos más brillantes en el desarrollo de los grupos sociométricamente desarrollados fue aquel en que se pudo demostrar que esa relación puede ser invertida: que el médico puede transformarse en paciente y el paciente en médico; que todo miembro del grupo puede transformarse en terapeuta de cualquier otro. Debemos distinguir, por lo tanto, entre el que en conjunto dirige una sesión y los "agentes terapéuticos". El agente terapéutico, en psicoterapia de grupo, no tienen por qué ser un individuo con un status profesional, un médico, un sacerdote o un consejero. En realidad, una persona que posee status profesional puede, por esa misma razón, resultar nociva para un determinado individuo que necesita ser atendido. (Moreno, 1967, pág. 25)

Esta base de la terapia psicodramática es clave para el trabajo con comunidades afectadas por catástrofes por cuanto permite realizar el empoderamiento de su propia salud mental, no solo a nivel de la acción comunitaria social, sino a nivel de la interacción individual de las personas afectadas, las habilidades de los sujetos puestas en juego con el psicodrama convierten a los mismos sujetos afectados en agentes de su recuperación de manera concreta, personal inmediata. Rompe además la verticalidad de la relación entre terapeuta y grupo, y convierte a todos los involucrados en agentes activos principales y fundamentales del proceso, generando nociones de dignidad, empoderamiento, y desarrollo de herramientas psíquicas, muchas veces ocultas hasta que el sujeto es expuesto al desarrollo sociométrico grupal.

En el momento que el ejercicio psicodramático comienza sus participantes pasan a ser nuevamente sujetos empoderados de sí mismos, la condición de víctima, de quien "recibe ayuda", de "enfermo" desaparecen, y el sujeto es capaz de asumir su propia recuperación e inclusive la recuperación de los otros, es por esto que Moreno planteará que el te- 
rapeuta debe acogerse a los postulados de "lo primero es el grupo, el terapeuta debe estar subordinado a él", "el terapeuta hasta el momento de surgir como líder terapéutico, es un miembro más del grupo"y finalmente "un hombre es el agente terapéutico de otro y un grupo el agente terapéutico de otro"; estos postulados implican que la misma comunidad generará las necesidades grupales que deben ser abordadas, no existe una imposición de aquello que el actor externo cree que se debe trabajar, sino que el grupo espontáneamente abordará aquello que le preocupa y le interesa, el terapeuta además necesita introducirse en el grupo, ser parte de, cumplir con el principio de un "no hacer en", sino "hacer con", que dirige la psicología comunitaria, y el tercer postulado implica que el grupo es para el otro, el fin de mi accionar, y la acción de todos, es el otro, recuperar el tejido de solidaridad y vínculo que se ve fragmentado por la situación catastrófica.

Para poder realizar este proceso de empoderamiento del sujeto y del grupo de su propia recuperación requiere una adecuada investigación sociométrica de grupo, que permita leer, entre otras cosas, los fenómenos de tele que aparecen en el grupo así como los estatus sociométricos de los participantes:

Para embarcarse en un acción conjunta, el equilibrio debe existir no solo en cada uno de ellos, sino también entre ello, formando así una unidad sociodinámica. Nuestra hipótesis principal, por lo tanto, consistió en la existencia de un factor hipotético, la tele, y en el grado en que el mismo actúa en la formación de agrupamiento, desde las díadas y los triángulos hasta los grupos de cualquier tamaño. (Moreno, 1967, pág. 27)

Este análisis permitirá gestionar las capacidades sociométricas de los participantes de la actividad grupal, y a base de estas permitir que el mismo grupo reconstruya las formas básicas de tejido social que la catástrofe desintegró, y a base de estas potencialidades dar los primeros pasos en la formación de nuevos agrupamientos donde las herramientas psíquicas de los diferentes participantes puedan ponerse nuevamente al servicio de las necesidades personales y los fines comunitarios, garantizar además la libertad de la comunidad en el desarrollo de esta construcción del bienestar común.

El encuentro con la comunidad ya no se halla por lo mismo regulado por el control alienante, por la disciplina del albergue, sino por un espacio de libertad grupal basada en poder establecer acción dialógica con el sujeto que comparte la situación de la catástrofe, y a partir de ese encuentro generar redes afectivas, redes sociales, espacios de acción 
comunitaria, que reconstruyan el tejido social, que redignifiquen al afectado, que potencien a la comunidad, y que se con- viertan en herramientas de la salud mental y la liberación.

\section{CONCLUSIONES}

La psicología social, de la liberación, política o comunitaria ha desarrollado un extenso corpus teórico que le permite brindar respuestas técnicas y prácticas a situaciones de emergencia y desastres, las mismas que se sustentan en una visión social de la crisis centrada en la lucha del sujeto por su liberación.

Las situaciones de crisis requieren aplicar el análisis dialéctico de la relación entre lo personal, lo comunitario y lo social, para a base de esto realizar una planificación que no cumpla solamente con el control funcional, sino que permita el empoderamiento de la comunidad de su propia salud mental y sus procesos de liberación.

El psicodrama es una herramienta fundamental para el psicólogo social por cuanto le permite generar los espa- cios de libertad necesarios donde el sujeto afectado por una catástrofe supere el estado de inhibición, se empodere de su propia salud mental, redignifique su vida, y reemprenda su proceso de liberación. Al mismo tiempo es un espacio comunitario donde el encuentro permite potenciar la dinámica comunal hacia los fines de trasformación de las comunidades oprimidas.

El psicólogo social no es un asistencialista o un interventor externo, tampoco aliena a la comunidad de su propia actividad y decisión, es un agente de la transformación social que lucha como un miembro más de la comunidad, con las herramientas que su formación profesional le ha brindado en la construcción de una sociedad mejor. 


\section{BIBLIOGRAFÎA}

Deleuze, G. (1995). Conversaciones 19721990. Valencia: Pretextos.

Martín Fernández, C. (2006). Psicología Social y vida cotidiana. La Habana: Félix Varela .

Moffatt, A. (1974). Psicoterapia del oprimido. Buenos Aires: Ecro.

Moffatt, A. (1982). Terapia de crisis. Buenos Aires: Búsqueda.

Moffatt, A. (1986). Psicoterapia existencial. Buenos Aires.

Montero, M. (2004). Introducción a la psicología comunitaria. Buenos Aires: Paidós.

Montero, M. (2006). Teoría y práctica de la psicología comunitaria. Buenos Aires: Paidós.
Moreno, J. (1961). Psicodrama. Buenos Aires: Emecé.

Moreno, J. (1967). Las bases de la psicoterapia. Buenos Aires: Hormé.

Pichon-Rivière, E. (1985/ 2006). Teoría del vínculo. Buenos Aires: Nueva Visión.

Pichon Riviere, E. (1999). La psiquiatría una nueva problemática. Buenos Aires: Nueva Visión.

Pichon- Riviere, E. (2006). Psicología de la vida cotidiana. Buenos Aires: Nueva Visión .

Vygotski, L. (1979/2009). El desarrollo de los proceso psicológicos superiores. Madrid: Crítica. 
